\title{
Homecoming: Exploring Returns to Long-Term Single Player Games
}

\author{
Noor Hammad \\ University of Calgary \\ Carnegie Mellon University \\ Pittsburgh, PA, USA \\ nhammad@cs.cmu.edu \\ Sowmya Somanath \\ University of Victoria \\ Victoria, BC, Canada \\ sowmyasomanath@uvic.ca
}

\author{
Owen Brierley \\ University of Calgary \\ Calgary, AB, Canada \\ owen.brierley@ucalgary.ca
}

\author{
Patrick Finn \\ University of Calgary \\ Calgary, AB, Canada \\ pfinn@ucalgary.ca \\ Ehud Sharlin \\ University of Calgary \\ Calgary, AB, Canada \\ ehud@ucalgary.ca
}

\author{
Zachary McKendrick \\ University of Calgary \\ Calgary, AB, Canada \\ zachary.mckendrick@ucalgary.ca
}

\author{
Jessica Hammer \\ Carnegie Mellon University \\ Pittsburgh, PA, USA \\ hammerj@andrew.cmu.edu
}

\begin{abstract}
We present an autobiographical design journey exploring the experience of returning to long-term single player games. Continuing progress from a previously saved game, particularly when substantial time has passed, is an understudied area in games research. To begin our exploration in this domain, we investigated what the return experience is like first-hand. By returning to four long-term single player games played extensively in the past, we revealed a phenomenon we call The Pivot Point, a 'eureka' moment in return gameplay. The pivot point anchors our design explorations, where we created prototypes to leverage the pivot point in reconnecting with the experience. These return experiences and subsequent prototyping iterations inform our understanding of how to design better returns to gameplay, which can benefit both producers and consumers of long-term single player games.
\end{abstract}

\section{CCS CONCEPTS}

- Applied computing $\rightarrow$ Computer games; $\bullet$ Human-centered computing $\rightarrow$ Interaction design process and methods; User centered design.

\section{KEYWORDS}

long-term single player game, autobiographical design, Pivot Point

\section{ACM Reference Format:}

Noor Hammad, Owen Brierley, Zachary McKendrick, Sowmya Somanath, Patrick Finn, Jessica Hammer, and Ehud Sharlin. 2021. Homecoming: Exploring Returns to Long-Term Single Player Games. In CHI Conference on Human

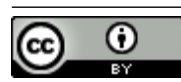

This work is licensed under a Creative Commons Attribution International 4.0 License.

CHI '21, May 8-13, 2021, Yokohama, Japan

(C) 2021 Copyright held by the owner/author(s).

ACM ISBN 978-1-4503-8096-6/21/05.

https://doi.org/10.1145/3411764.3445357
Factors in Computing Systems (CHI '21), May 8-13, 2021, Yokohama, Japan. ACM, New York, NY, USA, 13 pages. https://doi.org/10.1145/3411764.3445357

\section{INTRODUCTION}

Long-term single player games such as Assassin's Creed or The Elder Scrolls give the player control of a single character, which they can guide through a series of story missions and, often, within an open world. These games promise players an immersive play experience, along with gameplay systems that require this longterm investment to fully master.

Robust single-player experiences offer many advantages for players. For example, single-player games neither require the coordination costs of organizing a group of friends to play, nor expose the player to potential harassment from strangers online. Deep and immersive systems encourage replay, which gives players more pleasure for the money already invested in the game. To amplify the value of long-term single player games, game companies support continued engagement through post-launch content. Postlaunch content extends the storyline and gameplay of the base game through downloadable content and expansion packs. These are often substantial releases that add dozens of hours of gameplay to an already large game, and as a result require lengthy development times. The Witcher 3: Blood and Wine, for example, released over a year after The Witcher 3: Wild Hunt.

Post-launch content incentivizes players to return to games months or years after release, when they may no longer be actively playing. Players may also return to games because they were interrupted in their original play by life circumstances, such as having a child or moving to a new city. In both cases, return is distinct from replay, because players are picking up a game already in progress. They do not have access to the game's tutorials or scaled introduction of systems, and they may be picking up a complex narrative in the middle. Meanwhile, game systems do not recognize that the player is returning to rather than continuing gameplay uninterrupted, and hence do not accommodate their circumstances. 
Our work seeks to improve the return experience for long-term single player games. While players are frequently incentivised to engage in return gameplay, this experience has not previously been studied. In our work, we investigate the return experience from a player perspective through autobiographical design. First, the first author, referred to as the Player-Designer, returned to four longterm single player games she played extensively in the past, while recording her experience in an audio and written journal. We then analyzed and reflected on this data, revealing a consistent pattern to return play. This pattern hinges on the Pivot Point, a moment that reminds the Player-Designer of her expert-self and positively propels the return experience forward. Finally, we explored how to manifest the Pivot Point in design through both low- and highfidelity prototypes by the Player-Designer.

Our contribution to the HCI community is an exploration of a novel design space. We describe the details of the autobiographical journey of returning to four beloved long-term single player games and share the subsequent design process. We then share what we learned from this process, including a reflection on the limitations of our ideas we encountered in practice. We explain why combat played an important role in pivot facilitation in the games we examined, speculate on how the Pivot Point relates to existing literature, and position the Pivot Point as an integral part of the return phase in the journey of play.

\section{RELATED WORK}

To understand the challenges of returning to long-term single player games, we look both at HCI research and at game industry practices. First, we define what we mean by long-term single player games; we then explore transitions in technological experiences within $\mathrm{HCI}$; finally, we look at the ways that games address the problem of return, typically framed as an issue of retention.

\subsection{Long-term Single Player Experiences}

We define long-term single player games as a one-player experience designed for engagement of up to hundreds of hours, wherein the player is required to invest this time of gameplay into narrative, inventory management, combat skill-acquisition and character growth to significantly progress in the game.

Long-term engagement in games offers benefits different from short-term play. Engagement for extended periods of time can indicate player's satisfaction with the game, support long-term play objectives, increase the reliability of the game and the pleasure of being part of a game's community [17]. Long-term games can extend beyond entertainment to encourage physical activity over time [28], and allows players to become more literate in the game's language and its complexity [22]. Long-term engagement also fosters mastery or expertise $[3,16]$. Gee identifies the "cycle of expertise" that games adopt to cultivate iterative skill growth, and long-term engagement can further deepen that growth due to the greater exposure [23].

Single-player games, as opposed to multi-player games, are experiences designed just for one person. Chen and Keating et. al both identify that massive online games depend on the ability of the group to coordinate their efforts to achieve success, and that coordination is a complex task $[6,29]$. While the social nature of gameplay can increase retention, it can also give rise to players moving on from the game [11]. Single-player games do not require group coordination and thus success in the game does not depend on how well a group of players work together in-game.

Harassment in multi-player games have been shown to lead female gamers to play alone or anonymously [34], or even to withdraw from the game [18]. Single-player games, in which the player does not have to interact with other players, can be a haven away from harassment. Single-player games also foster companionship and rich social experiences normally found in multiplayer experiences through in-game companions [14].

The extremely profitable games-as-a-service model has influenced the single player landscape as well, with loot boxes, microtransactions, season passes and story-based downloadable content increasingly utilized as monetization and retention tactics. Single player games, despite regularly selling millions of copies and topping monthly sales charts [4], are now driven towards cultivating continued player engagement and player investment through substantially greater amounts of in-game content [30] and the implementation of role-playing mechanics $[10,21]$. The ubiquity of long-term single player games, and the post-launch content practices, means a rise in complexity of the game experiences developers are asking players to engage, and re-engage in; complexity that the player alone, without an online group, is taking on.

\subsection{Transition Experiences}

The return to a game is a type of transition. There is a growing body of work in HCI research concerned with state change and transitions in technological experiences $[24,31]$. One of our primary inspirations was Knibbe et. al's exploration of the experience of exiting virtual reality (VR), which sought to understand the momentary experience of exiting a VR environment and the subsequent transition back into the real world [31]. Knibbe et. al detail participant stories of their "moment of exit" and speculate on the design of future VR applications that could leverage the moment of exit to extend the experience beyond the headset. Transition theory has also been applied in other domains of HCI research, such as cyborg wearables [24]. Here, Hammad et. al explore creating protocols to optimize the transition to and from a cyborg state using performing arts techniques, which helped ease the transition for the performers in the study.

In games research, transitions have primarily been studied in two ways: the transition into a game, and the transition to an immersed state. Cheung et al, describe the "first hour of experience" that is critical to engagement, as games are easily abandoned if they are not not interesting enough [7], or abandoned if they are too difficult [39]. Should players feel the first hour of experience is too difficult or confusing, such as having a complex crafting menu, then they can feel frustrated and abandon the game. However, Cheung et. al's research focuses only on the first hour of experience with a new game, and does not consider the context of the first hour of a return. Andersen et. al have examined the impact of game tutorials on learning and engagement, indicating that players could benefit more from in-game tutorials in a complex game [1]. Long-term single layer games are complex, yet there is no work on what the return to a game is like without those early tutorials. 
In prior work on immersion, Emmerich et all provide a study of the use of compelling in-game companions, both human and non-human, as a means to induce, sustain and deepen immersion, with an aim of providing insight into how to improve the play experience through better companion design [14]. In Game Studies, Carl Therrien's description of two types of immersion (illusory and engaging) and three kinds of immersive experience (psychological, flow-state and fictional) are used to describe blended engagement models [46]. Iacovides et. al further our understanding of breakthroughs and breakdowns in games by examining what strategies players employ to achieve progress, yet their work stops short of extending the exploration to long-term single player games [26]. We learned how immersion is achieved and sustained through game design from these works, but we still do not know how it is affected upon returning to a game and what game design mechanisms there are to support this transition.

\subsection{Continued Player Engagement}

Prior work on long-term engagement with games has focused on multi-player games, as well as on the concept of retention rather than return. In the large-scale multiplayer game space, including MMOs, retention strategies are fundamental aspects of game design used to support continued player engagement and monetization of the service. Strååt and Verhagen used longitudinal methods to identify where breakdowns in retention may occur in Tom Clancy's The Division [44]. Meanwhile, Debeauvais et. al analyze retention mechanisms in World of Warcraft by sampling over two thousand World of Warcraft players across the globe, identifying factors such as social connection, in-game reward, and achievement structures as particularly potent retention strategies in certain demographics [11]. The questionnaire used metrics such as hours of play per week, length of total play time and the stop rate, which is the ratio of respondents who stopped playing World of Warcraft and then returned to the game. The stop rate in their sample was $77 \%$, meaning only $23 \%$ never stopped playing. Despite identifying that players do stop playing for some period of time before returning, there is no work studying what that return is like.

While we have not found any research on return in long-term single player games, we observe that some games already use design strategies to support both ongoing gameplay and return. For example, The Witcher 3: Wild Hunt attempts to catch up the player on what they did using videos and plot summaries in the loading screen, akin to "Previously on..." segments for TV shows. This approach only captures the past narrative events, and is not widespread in long-term single player games [19]. Some games, like early Assassin's Creed titles, support player interaction in the loading screen "memory corridor" by giving them control of the playable character in an empty space [48]. This can work as a limited mechanical warm-up, although there are no objects to interact with in the space. These approaches can capture specific elements of the experience, but not the overall gameplay factors the player will need to continue progressing upon return.

Based on this literature, we explore the process of returning to long-term single player games through the lens of managing a technological transition. We seek to understand how players can return to game saves that took dozens of hours to cultivate, and how designers can better support the process of return.

\section{METHODS}

To explore what it is like to return to a long-term single player game, we were inspired by first-person research practices in HCI. Methodological approaches such as autoethnography and autobiographical design have gained traction in the HCI research community [32, 33, 36]. In autoethnography, a fieldworker's experience is investigated together with the experience of the observed social actors in an ethnographic study [33]. This produces a reflexive, first-person recount of the conducted ethnographic work wherein the fieldworker exposes their personal experience to the reader. Autobiographical design, on the other hand, is design research drawing on extensive, genuine usage by those creating or building the system [36]. By engaging in personal use, the experience of the researcher at the center of an autobiographical design inquiry is embodied in the subsequent system iterations and design lessons learned [35, 37].

Both methods acknowledge and emphasize the first-person role in a research activity, however autoethnography requires the observation of other social actors, and our work is concerned with single-player games. While we take inspiration from autoethnography, particularly in producing rich, first-person reflections, we chose autobiographical design as the overarching method. With this approach, we can gain an experiential understanding of returning to long-term single player games to inform the design of better return experiences.

Our work here benefits from previously identified autobiographical design advantages. First, autobiographical design cultivates a rich, personal, and experiential data set resulting from self-usage that can be leveraged in the design process. We receive intimate insight into the process of returning to a once beloved and expertly played single-player experience, through the lens of an expert gamer and designer. Furthermore, autobiographical design has been championed as being particularly effective at helping the designer to repeatedly reflect upon their design decisions throughout the process of innovation [37], as Mellis et. al demonstrated in their first-person investigation of high-tech DIY practice [35]. In our case, the personal insight gained from a designer's perspective helps us understand what it means to return to our games of focus, which in turn informs our understanding of the novel design space. Thus, through autobiographical design, we take our first steps into the realm of designing better returns to long-term single-player games. We frame this as an exploratory design endeavour for defining a new space within games production and research.

\subsection{The Player-Designer}

At the center of the autobiographical design inquiry is the PlayerDesigner, an avid gamer who plays long-term single-player games on a regular basis. The Player-Designer is a member of the research team with experience in HCI research and design. The games chosen for our work had the Player-Designer cultivate expertise through dozens of hours of gameplay, and, crucially, were ones she had a desire to return to independent of the study. In other 
words, the Player-Designer expressed a "genuine need" for using the software.[36].

The Player-Designer's autobiographical design journey consists of two stages: a Gameplay stage, in which she experienced returning to her last saved progress in a series of long-term single-player games, and a Design Exploration stage, where she iteratively prototyped, on paper and digitally, ways to improve returning based on her own experience. This two-phase approach informs our choice of the "Player-Designer" label; it captures the play-then-design exploration from the first-person perspective. Together, the two phases combine to form a single autobiographical design journey, however the journaling approach in the gameplay stage is inspired by autoethnographic reflections, such as Amon Rapp's first-person recount of playing World of Warcraft [41].

3.1.1 Gameplay Stage. Here, the Player-Designer revisited four games she had played extensively in the past; Assassin's Creed: Odyssey (Odyssey), God of War, Horizon: Zero Dawn (Horizon) and The Witcher 3: Wild Hunt (Witcher) [20, 40, 42, 45]. The four chosen games were not developed internally by the research team; these games are commercially available and are representative of the long-term single player games we are examining and aiming to design for. While this means the self-usage the Player-Designer engaged in was not of a system built by the research team, as is specified by autobiographical design, these games were selected because the Player-Designer had played many hours in each game and because she has a strong desire to continue playing them. We note that each game was played for over forty hours, a consequence of the time required from the Player-Designer to reach high-levels of expertise.

The games were returned to in the order they were last played: Odyssey (two months before the study date), God of War (one year), Horizon (two years), and Witcher (three years). The range of times when the games were last played were by-products of when the Player-Designer originally stopped playing the game. That this range of times played is spread out as such is a coincidence rather than a condition of selection.

The returns were in the form of one play session for each game that was three and a half hours in length. While short within the context of long-term play, it is a sizable play time within the context of post-launch content (typically 4-12 hours). The length allowed the Player-Designer to complete quests, fight enemies and traverse the world while keeping the dataset manageable for analysis.

During the play sessions, the Player-Designer created artifacts in the form of in-situ audio logs and retrospective written journals. The audio logs captured real-time thoughts during gameplay, and the journals were for post-game reflection. The dataset was supplemented with screenshots and gameplay video captures. The collective data sources were qualitatively analyzed using thematic coding. First, the Player-Designer coded the study material using bottom-up techniques. The 'Pivot Point' first emerged as one of these codes. All codes were reviewed regularly with the study team and analytic memos created. During this process, the Pivot Point emerged as a central theme. Existing codes were then grouped into Pre-Pivot, Pivot Point, and Post-Pivot stages. A second, orthogonal grouping of codes reflected gameplay factors that the Player-Designer was exposed to in the play sessions: Narrative (quests, characters, lore), Controls (combat, stealth, traversal), UI (HUD, inventory, menu systems) and World Navigation (geography, in-game locations). This gave us a breakdown of how gameplay was affected by the return and the role it played in the Pivot Point. Finally, we derived themes of the emotional, cognitive and mechanical impact on these affected gameplay factors in each of the three stages. We note that our descriptions of the Player-Designer's engagement in the gameplay stage are taken from the analysis of the journals and were not measured.

3.1.2 Design Exploration Stage. Following the reflection and analysis of the returns, The Player-Designer explored how to improve her return experience, and how to facilitate the Pivot Point, through low- and high-fidelity prototyping. First, the Player-Designer created sketches for each game aimed towards optimizing her return experiences and Pivot Point occurrence. This early sketching process used a ten-plus-ten prototyping approach [5]. The resulting sketches were then reflected upon by the Player-Designer, and the themes found in the sketches served as the first iteration of the final design goals described in section 5. The Player-Designer continued refining the design goals through low-fidelity prototyping and discussion with the research team. This iterative design-feedback process concluded with the Player-Designer implementing a digital prototype in-game to better understand how the created sketch designs that captured the final design goals translated to gameplay. The designs created by the Player-Designer are drawn directly from her experiences playing the four return experiences from the gameplay phase. All design features created in this phase can be supported by the four games we explored.

As our methods demonstrate, this work is both a piece of autobiographical design and a product of a research team. Desjardins and Ball [12] address the contributions other researchers offer in the design process as a potential source of tension in an autobiographical design inquiry, yet emphasize that despite the involvement of external actors, the Player-Designer is still the authority in her own process. As such, the paper is written in the first-person plural voice and uses third-person singular when referring to the PlayerDesigner and her activities.

\section{THE GAMEPLAY STAGE}

The experience of returning to a game involved struggles, but also breakthroughs in reconnection. Over the course of 15 hours of play across the four games, the Player-Designer had a range of interactions with game systems, gameplay encounters, and emotional experiences. However, we identify a key organizing concept: a Pivot Point that changes the lived experience of return. We define this Pivot Point as a spark of recognition in gameplay, a 'eureka' moment for the Player-Designer wherein she felt synchronized with the mechanics and her once expert self's behaviors, thus offering a glimpse of why she was willing to invest so much time in these games in the first place. The Pivot Point emerged consistently in all four return experiences. Before the Pivot Point, the PlayerDesigner struggled to interact with the game and felt frustrated about it. Within the set of games played during these experiences, the Pivot Point occurred during a combat encounter approximately three hours into each play session, giving the Player-Designer an exhilarating moment of competence. This led to a much more positive 


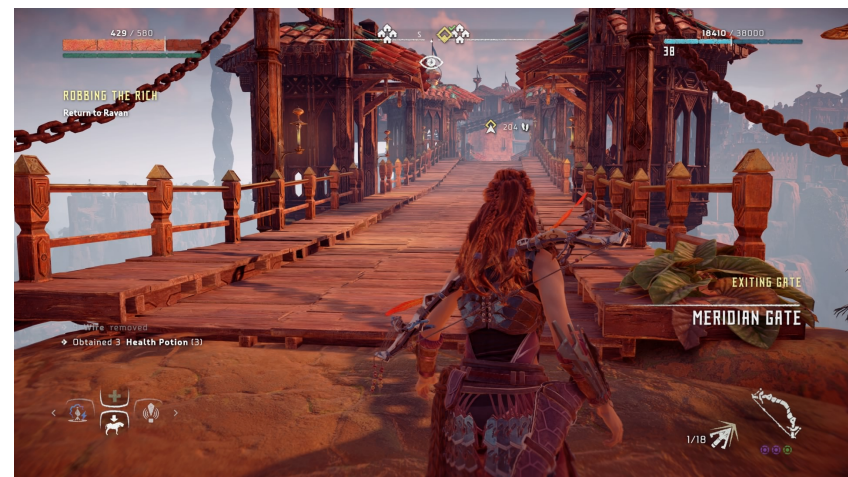

Figure 1: The Player-Designer entered Horizon: Zero Dawn with a quest (top left) already active

player experience post-pivot, even as many of the same struggles from the pre-pivot experience remained. To share the return struggles and breakthroughs as well as the Pivot Point, we highlight the pre-pivot, Pivot Point and post-pivot experiences below with details drawn from the Player-Designer's journals and audio logs.

We evaluate the return experience using the three facets of mechanics, cognition and emotion, which is similar to the MDA framework [25]. As in MDA theory, we recognize the critical importance of evaluating the gameplay experience mechanically, dynamically, and aesthetically. We argue that the Pivot Point is a key mechanical, dynamic and aesthetic experience emerging from mechanical progression through combat as part of a trajectory to reclaim the former expert self.

\subsection{Pre-Pivot Experiences}

In each play session, the Player-Designer initially struggled with reconnecting mechanically, cognitively, and emotionally with her expert-level self. While the time away from each game was different, the length of the break seemed irrelevant to the Player-Designer's struggle.

4.1.1 Mechanical Incompetence. With regard to the mechanics and control of gameplay, the Player-Designer found her cultivated skill from the past inaccessible. She could not complete combat encounters with the same skill and finesse as before, and regularly lost battles. "Well... my first fight lasted a solid 30 seconds, that was not fun. I don't know how to fight this enemy" [Horizon]. In her journals, the word "rusty" was regularly used to describe the state of her combat ability. "I feel super rusty in combat, the controls were really difficult, I forgot how combat works. And trying to remember how to fight in real time (when enemies are attacking quickly) is really difficult" [God of War]; "I don't know all the controls, I don't know the rhythm, this game is all about combat rhythm and keeping track of all the indicators and when enemies (hit you) you and (knowing what) their abilities are is hard" [Witcher]. These issues extended beyond combat to stealth and traversal. In her Odyssey reflection, she shares that she "Sometimes accidentally attacked instead of sneaking." These issues were amplified because her previous saves were late in the games. Her playable characters were therefore high level, and the encounters fought were also

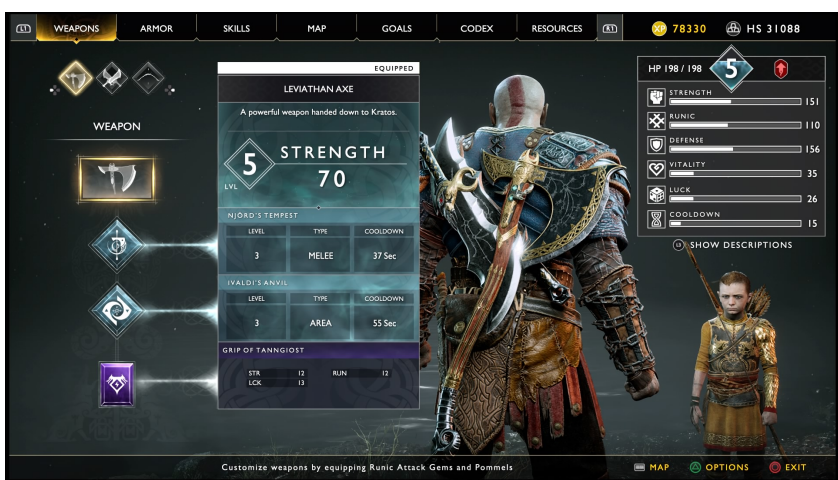

Figure 2: The menu system in God of War contained an overwhelming amount of information that was initially acquired over time

of a higher level, meant to challenge expert players. For example, "Control (and) combat issues were really magnified when (I was) fighting a lot of enemies," which is a technique used to scale up the difficulty of encounters in Odyssey.

4.1.2 Cognitive Load. Returning to heavily played, in-progress saves resulted in heavy cognitive load for the Player-Designer. First, to make meaning out of the game's events, the Player-Designer needed to remember the finer details of complex lore. Second, she had to make sense of her past self's decisions. She often entered the games with quests already in-progress, struggling to remember why she left it incomplete and what to do next (Figure 1). "I'm dropping into this game, like 40 hours in, and I don't remember why I left quests where I did, and where I'm supposed to pick up all of that information" [Horizon]. She also could not remember why her past self left valuable resources like skill points and better armor unused. "Why did I choose these weapons (over the higher level ones I also have)?" - [Odyssey]. She described these initial memory difficulties as "almost like getting dropped onto a moving treadmill" and "like a programmer revisiting their code after time away and wondering what their code meant" [Odyssey]. "I'm trying to recapture or get back into the state of mind that made me make those decisions, to actually get back into (that) state where I can play the game based on the choices I've already made." [Witcher] The greatest source of cognitive trouble, however, resulted from the in-game menus (Figure 2). Each game has complex menus for inventory, skill upgrades, quest logs, navigation, and lore details. These menus initially took time to learn how to operate, and the player was scaffolded in doing so by the game. Additionally, the items within them were populated over a long period of time. Returning to them all at once, she was overwhelmed by the sheer amount of content and options. In an attempt to help find her footing, and because she was so used to navigating these menus in each game with ease, the Player-Designer spent a considerable amount of time trying to unravel the menus. "This game always had so many layers (that you learn) as you go, and now I'm trying to re-learn all those layers that took time to learn in the first place." [God of War]. The phrase "too much reading" regularly appeared in the journals, "I don't think spending 40 minutes of your game time reading stuff is the most 
efficient way or fun way to spend time in a video game.. [ Witcher] "Just (trying to get) caught up on the narrative (using the quest $\log$ ), it's just a ton of reading. there's so much reading, I just spend so much time reading and not enough doing. And because I'm reading so much, it's not very engaging, I don't retain a lot of the information." [God of War]. The cognitive input from trying to read everything in the in-game menus was overwhelming, which proved to be emotionally difficult for the Player-Designer as well.

4.1.3 Emotional Overload. This combination of mechanical incompetence, inaccessible memory and overwhelming content resulted in feelings of stress, frustration, and embarrassment for the PlayerDesigner, to the point where she considered stopping playing, a major breakdown in the experience [27]. Mechanical incompetence contributed to the lack of enjoyment, "I'm just so rusty right now, and it's just not fun" [Witcher]. The feeling of embarrassment emerged from a lack of mechanical competence and led to the Player-Designer questioning how she ever reached expert skill in these games in the first place. "Goodness this is so... It feels like I've never played this game before." [God of War]. Attempting to unravel the menus was a source of extreme negative emotion. "Without question the most overwhelming part is those menus. The reason being, it's just so much stuff, it was so stressful and if I wasn't doing this for a play session, I would've probably stopped the game right there." [God of War]. "But the most overwhelming aspect, and something that would have definitely stopped me from playing is the menus. There's just so much reading, and I didn't want to do that anymore." [Witcher].

As the play sessions progressed, the negative feelings described above continued to plague the play sessions, but there were minor breakthroughs in combat and cognitive understanding that grew as the Player-Designer gained more exposure to the world through combat. "I'm slowly starting to use more and more of my arsenal of weaponry. So I'm starting to use more power-ups, use more of heavy attacks and things like that." [Odyssey]. These minor breakthroughs were few and far between until the Pivot Point, where the minor successes in combat accelerated to be a major breakthrough in the entire return experience.

\subsection{The Pivot Point}

Approximately two and a half hours to three hours into the play session, there was a particular combat encounter where the PlayerDesigner was more engaged mechanically, cognitively, and emotionally than ever before, resulting in the feelings of euphoria and excitement that carried forward the rest of the session. We frame this encounter as the Pivot Point, where the minor breakthroughs in reconnecting with the game were accelerated to a major shift. As a result, the largely negative experience of gameplay return "pivots" to be largely positive. In God of War, for example, the encounter was with a high-level "boss" known as a Valkyrie. "I just beat a Valkyrie! Combat felt SO good there. That's the first time combat clicked, I think. There is still a lot that I need to learn...but I'm feeling a lot better. That was awesome." [God of War]. The Pivot Point is where the previously inaccessible expert self's skills were momentarily enabled and served as a moment of synchronization with the Player-Designer's former expert self, mechanically, cognitively, and emotionally. "I just had another fight, a big fight, and I'm having a lot of fun, I didn't lose any health. (I feel like) I'm finally back in the swing of things, and I love this game, I'm back to actually enjoying it. It took (three) hours but I'm back in." [Witcher].

\subsection{Post-Pivot Experiences}

Given how much she was struggling with the return, the mechanical competence and cognitive focus that was channeled in the Pivot Point encounter was a hugely positive experience for the Player-Designer, highlighting that her expert skill was in fact in reach. Being skilled in combat, and cognitively and emotionally unburdened in the Pivot Point encounter, resulted in a feeling of euphoria that led her to becoming motivated to find that expert self again. "I remembered how much I loved this game when combat finally clicked and I did more quests (as a result). (at first it) felt odd, the second half (of the play session) felt more natural and was more fun." [Odyssey]. The pre-Pivot Point struggles were such that the Player-Designer considered quitting God of War and Witcher. The post-pivot experience completely changed the experience for the Player-Designer in these games, "I genuinely wanted to stop playing because I felt stressed and lost. (But after defeating the Valkyrie) I started remembering why I fell in love with this game. By the end, I didn't want to stop playing"[God of War]. "It started out as being extremely overwhelming, and stressful, and then turned into remembering why I fell in love with this game, and just loving the experience and having fun to the point where I just didn't want to stop the play session. It started getting really, really fun, and I started seeking out these (combat) encounters." [Witcher]. This motivation-focused phenomenon's influence on the experience, and the elation that emerged from it showcases the power of this spark of recognizing the previous self that is the Pivot Point.

The Pivot Point is not the point where the Player-Designer's entire previous expertise returned for the rest of the play session, but rather where she began enjoying the experience again despite not being the full expert yet, and that her expert knowledge of the game can be recovered. The post-pivot experience featured some persistent combat difficulties, as the Player-Designer still hadn't completely "shaken off the rust"; "I just (lost) in combat again. But that's okay, I'm having fun." [Horizon]. The Player-Designer did not even engage with some entire sub-systems. "I didn't have the time (in the play session) to change my armor or weapons, so that's something I didn't entirely adjust to" [God of War]. The difference was less in skill than in the player's experience of her skill. "(Playing this game) is feeling good, even though it's difficult, and I don't think I'm back at the level of skill I was a year ago, but now it's starting to click, it's fun and I'm learning as I go instead of just being super confused...I don't feel as lost anymore" [Witcher]. The Player-Designer observed that after the Pivot Point, she was more mechanically competent (albeit not at expertise level), more eager to seek out combat encounters and to engage in all of the game's systems, and emotionally attached to the experience again. The mechanical, cognitive, and emotional aspects of the pre- and post-pivot experiences are captured in table 1.

\section{DESIGN EXPLORATION STAGE}

The experience of return to the four games illuminated a range of barriers, such as overwhelming amounts of text to process, 
Table 1: Summary of Mechanical, Cognitive and Emotional Experiences in the Pre-, Pivot Point and Post-Pivot Stages

\begin{tabular}{|l|c|c|c|}
\hline & Pre-Pivot & Pivot Point & Post-Pivot \\
\hline Mechanical & $\begin{array}{c}\text { Incompetent in combat and stealth, } \\
\text { struggling with high-level enemies }\end{array}$ & $\begin{array}{c}\text { expert control, } \\
\text { defeating difficult enemies } \\
\text { with finesse }\end{array}$ & $\begin{array}{c}\text { Requiring more combat } \\
\text { exposure to fully } \\
\text { regain expertise }\end{array}$ \\
\hline Cognitive & $\begin{array}{c}\text { Confusion due to difficulty remembering quest } \\
\text { progress and gameplay decisions. } \\
\text { Informational overload from } \\
\text { menu reading }\end{array}$ & $\begin{array}{c}\text { Unburdened by the pre-pivot } \\
\text { cognitive issues, entirely focused } \\
\text { on the combat encounter }\end{array}$ & $\begin{array}{c}\text { No longer as confused, } \\
\text { Emotional }\end{array}$ \\
\hline Overwhelmed, stressed, embarrassed, frustrated & $\begin{array}{c}\text { Not self-conscious, unburdened by } \\
\text { emotional difficulties from pre-pivot. } \\
\text { Synchronized with former self }\end{array}$ & $\begin{array}{c}\text { Feeling powerful, } \\
\text { competent, curious and eager } \\
\text { to rediscover full expertise. } \\
\text { Motivated to keep playing }\end{array}$ \\
\hline
\end{tabular}

challenges with recall of controls, and frustration with the gap between memory and execution. In theory, each of these barriers could be addressed separately. However, the Pivot Point provides a more streamlined focus for our design. Instead of generating designs for each sub-system of the game, we could design toward inducing a Pivot Point experience without the associated frustration of pre-pivot play. Rather than ensuring that every gameplay factor is accounted for in the design to guarantee full expertise recovery, the goal is to motivate the Player-Designer to keep going to find that expert self once again by facilitating the mechanical, cognitive and emotional spark.

The reflections and sketch iterations yielded two initial design goals: identify and minimize the negative emotion leading up to the Pivot Point, and focus on the primary driver of action and skill progression, which in the games we examined is combat, to actually facilitate the Pivot Point

\subsection{Design Goal 1: Minimize Negative Emotions Prior to Pivot}

It was clear that while the pre-Pivot Point experience was largely negative across the MDA spectrum, there were instances where the Player-Designer was so overwhelmed that she considered quitting the game. If the player is to be affected by the Pivot Point, they must keep playing until they reach it. We therefore identified these moments as a first target for design.

Our data shows that all incidents of wanting to quit the game were associated with inventory management or navigating the in-game menus. A commonality that emerged in every return experience was that the Player-Designer's attempt to fully understand the in-game menus resulted in feelings of stress. The impulse to read and re-engage through the in-game menus is understandable, as they are an essential component of our games in question, are revisited often during gameplay, and were once easily navigated by the Player-Designer. However, instead of serving as an opportunity for the Player-Designer to re-immerse in the gameplay world, the result is an information overload that was neither conducive to re-engagement nor any substantial positive feelings normally associated with her experiences in these games. Andersen et. al have also suggested that players learn more by playing than by reading text [1]. In normal play, in-game menus are accessed "on-demand" and give information as needed in the moment, not out of context.

The content in these menus are a result of dozens of hours of gameplay investment, and an active player recognizes how to make them part of the play experience. When they are being navigated in a return experience, however, written game materials appear contextless and overwhelming. As James Paul Gee highlights, "people are quite poor at understanding and remembering information they have received out of context or too long before they can make use of it" [23]. Hence, to minimize the negative emotion in the pre-pivot, we can address the use of inventories and menus. By delaying menu engagement until after the Pivot Point, the positive emotional experience of post-pivot play could buffer the negative experience of overwhelm.

\subsection{Design Goal 2: Focus on The Primary Driver of Skill Growth to Facilitate the Pivot Point}

The Pivot Point occurred when the Player-Designer's mechanical and cognitive focus were reawakened in a singular combat encounter. Combat is the source of progress towards the Pivot Point in the games we examined, and so by isolating combat as the main driver towards the Pivot Point, we are capitalizing on its pivotal role in facilitating the Pivot Point. However, the Pivot Point combat encounter was not the first encounter the Player-Designer engaged in. In all four games, it was only one in a series of combat sequences the Player-Designer was engaging in throughout the play session. The early combat engagements the Player-Designer underwent were not successful and her skills were "rusty", as she describes it. There is an evolution from her early combat encounters to the Pivot Point, implying that multiple exposures to combat were necessary to reach it. In the context of the games we returned to, by focusing on iterative exposure to combat, we can isolate and optimize the efficiency of when the Pivot Point is reached.

\subsection{Design Goal 3: Emotionally and Cognitively Align With Expectations of The Experience}

Cheung et. al highlight how reconciling player expectations with a game in the first hour can help set the tone for the experience or cause the player to abandon the game [7]. The pre-pivot cognitive load was partly due to a mismatch of expectations between the 


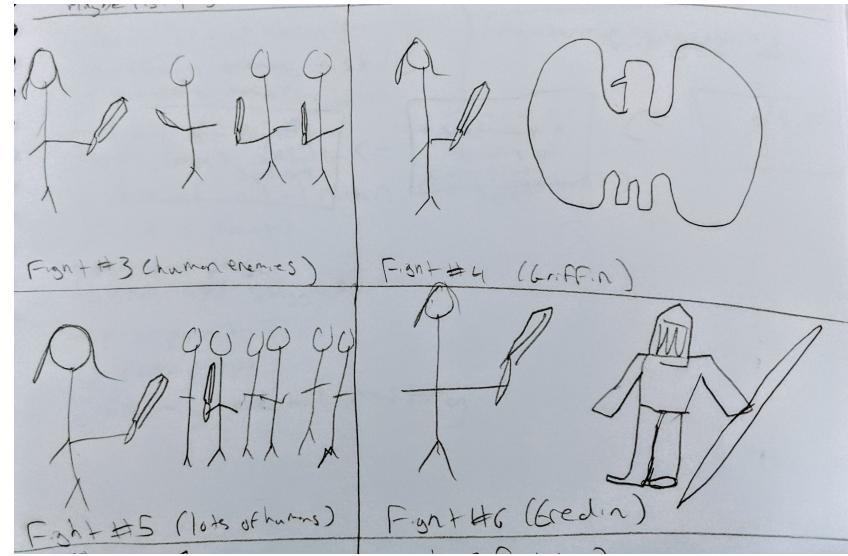

Figure 3: Practice arena sketches against level accurate enemies used in the final storyboard of the design exploration stage

experience the Player-Designer remembers, and the one she was met with. Emotionally, the Player-Designer felt disconnected from the love she had for the games. With this design goal, we want to contextualize the designs within the game world so as not to further exacerbate the mismatch of expectations, and leverage existing emotional connections to the games to better align with them as well. When designing for inventory and combat in Witcher, for instance, we must ensure that the experience still feels like Witcher, and not something that is a further mismatch with what the PlayerDesigner is expecting, or remembering, Witcher to be like.

Subsequent prototype iterations led to hands-off, "practice arena" style combat scenarios, where enemies are not scaled down, in lieu of tutorials that hold the hand of the player (Figure 3). The prototypes also accounted for the "dropped on a moving treadmill" feeling by disabling active quests. The Player-Designer also prototyped using familiar environments and UI elements to help facilitate the Pivot Point, and with emotionally resonant elements like beloved characters and music to align with the emotional expectations of the experience (Figure 4).

\subsection{High-Fidelity Prototyping}

The final step of the Player-Designer's autobiographical prototyping used in-game modifications, or "mods" to create, demonstrate and reflect upon more dynamic experiences of the Pivot Point. Mods rework the assets and code of a game to modify or extend the content [13]. For her high-fidelity designs, the Player-Designer used The Witcher 3: Wild Hunt modding tools, along with the communitybuilt quest creator Radish. Witcher was selected because it was the only game available on PC with extensive mod support at the time of the autobiographical design journey execution. The PlayerDesigner designed the prototype in the form of a playable quest, informed by the paper prototypes adhering to the design goals. Quests, and the design goals the quest follows, are common to all of the games in this study, as well as to other long-term single player games. This design choice means that we can extend our designs beyond the game used for modding, as more community tools

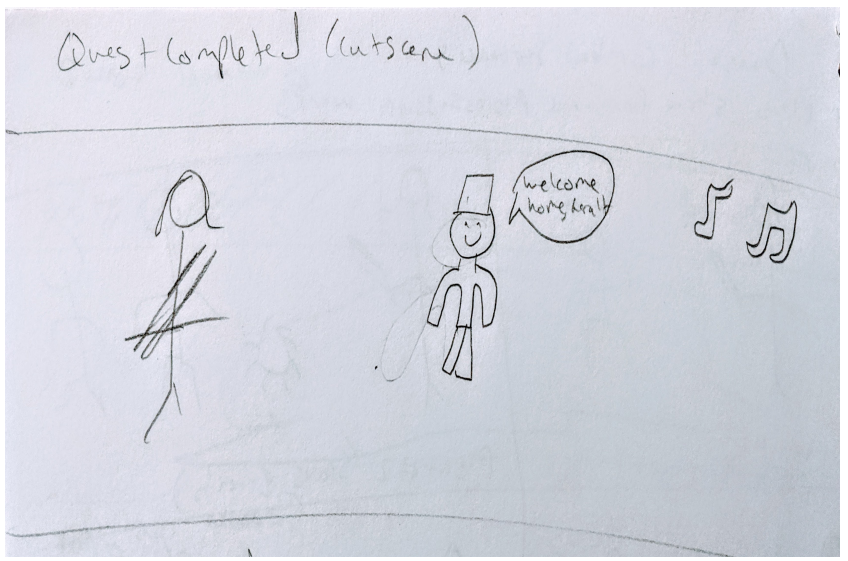

Figure 4: A sketch utilizing beloved characters and music in The Witcher 3: Wild Hunt

become available. While the mod was developed for The Witcher 3: Wild Hunt, the design features implemented are not specific to The Witcher 3. At the time of conducting this research, we only had access to the modding tools for The Witcher 3, but all proposed design features are common to all four games and the long-term single player game experience we defined.

The return mod consists of four parts that address the design goals and showcases the designs the Player-Designer sketched and storyboarded.

5.4.1 Part 1: Waking Up - Setting Expectations. The start of the mod has the playable character, Geralt, waking up, confused, and asking where he is. He finds himself in his home at Kaer Morhen (Figure 6), being greeted by his adopted daughter, Ciri. Ciri then guides the player throughout the experience to help Geralt get his bearings in his home.

This starting sequence serves two purposes, with the goal of aligning with player expectations (goal 3): introduce the player at a point meant for optimizing the return process without any active quests, while capitalizing on existing emotional attachment to the world and characters. Rather than arriving in the middle of an existing quest, this introduction and more insulated setting help reduce the cognitive load found in the pre-pivot state. Moreover, the emotional attachment is being used to help remind the Player-Designer of her connection to this world, and helps make the experience feel like Witcher.

5.4.2 Part 2: Don't Worry About It - Mitigating Inventory Surfing. To attempt to reduce the negative experiences of inventories and menus (goal 1), the Player-Designer designed a feature that outright disabled inventory access. In practice, this felt extremely aggressive, and actually conflicted with the goal of aligning with player expectations, as inventory engagement is a major element of gameplay. The Player-Designer iterated on this initial design with a pop-up adhering to the game's UI that asks if the player is sure they want to access the menus at this time (Figure 5). The Player-Designer then adjusted the text to be less of a warning, and more of a suggestion that focusing on the inventory is not needed to progress right now 


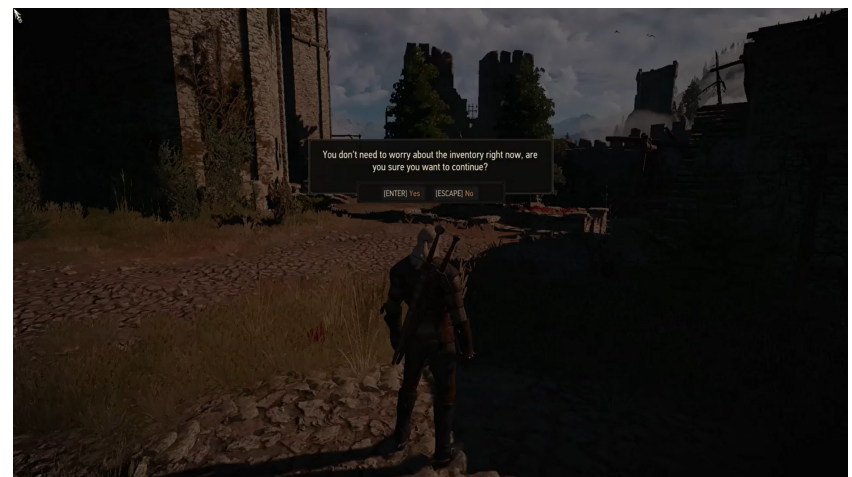

Figure 5: The pop-up dialog in the high-fidelity mod to mitigate menu reading

("Don't worry about this for now"). This suggestion is effective as a reassurance that the menu will not be needed in the quest.

5.4.3 Part 3: The Combat Maze - Combat-Driven Progression. The bulk of the quest is combat sequences (goal 2). As in the sketch prototypes, the Player-Designer created combat encounters with level-accurate enemies, but without the use of scaling techniques such as mass enemy attacks. These combat sequences are found around Kaer Morhen. They are placed such that they lead the player to the main gate that lets them exit back into their previous save once the quest is complete, almost like a maze. Four encounters were created featuring a range of enemy types. The final encounter is a "boss battle" against a gryphon, recreating a pivotal battle from the start of the Witcher storyline.

While the combat encounters were always meant to be representative of real combat found in the game, the Player-Designer iterated on the sketched designs to include more iconic enemies and bosses rather than just common enemy types found in the game world. This serves a similar purpose as the intro scene in terms of reigniting the emotional attachment to combat moments that feel representative of the game.

5.4.4 Part 4: Welcome Back - Exiting Prototype. After defeating the gryphon and reaching the main gate, the final scene of the quest plays. With Kaer Morhen in the background, Geralt stretches and "shakes off" the rust. Ciri stands in front of him, leading him out of the castle and back into the game.

As a parallel to the starting scene, this final sequence serves as an end to the quest and the Pivot Point-based return optimization. When prototyping this scene, the Player-Designer iterated on the interactions and behaviors of the characters to emphasize the "I'm back" feeling associated with a successful Pivot Point encounter. The Pivot Point always led to a feeling of euphoria, hence this scene aims to capitalize on that euphoria while also directing the momentum towards the rest of the game.

\section{DISCUSSION}

The autobiographical design journey the Player-Designer underwent had her return to four beloved long-term single player games to continue playing, reflect on those returns and center the subsequent design process on the Pivot Point. Here, we share the lessons

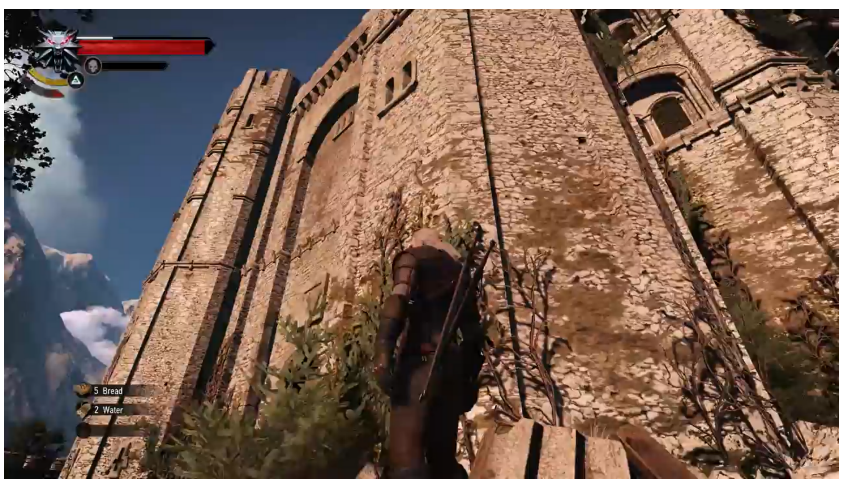

Figure 6: The high-fidelity mod takes place at Kaer Morhen, an iconic location in the The Witcher franchise

we learned from the autobiographical design process, speculate on what the Pivot Point is and what it means for the future.

\subsection{Learning From The Design Explorations}

We used the design explorations not as a method to directly produce working software but rather as a tool to learn more about the design space and what successfully designing for the Pivot Point entails. Through the Player-Designer's early iterations, we learned of the importance of setting expectations when designing for the Pivot Point such that we minimize cognitive and emotional dissonance. The high-fidelity prototyping through modding proved to be an important addition to the design process, as it allowed the PlayerDesigner to test how the designs were translating to gameplay and interaction.

6.1.1 Mitigating Reading in The Menus. While we believe the design goal of reducing negative emotions implemented as mitigating reading will help in Pivot Point facilitation, in practice it was a hard goal to execute well. The disabling of menu access was an aggressive measure, and, while a bit more nuanced, the pop-up dialog is also not ideal as it implies a sense of "incorrectness" to attempted menu access. The instinct to utilize the game menus in a pre-pivot state is understandable because of previous ease of navigation. Moreover, the menus are essential to fully experiencing the game and regaining expert skill. Delaying menu access may not be the right approach.

The issues the Player-Designer encountered with the menus were not due to the menu navigation and access, but rather because she felt the urge to re-read everything and tinker with her inventory. She was driven to understand all of the menus because she knew them so well at one point, and as a result felt that understanding them again would be key to reaching expertise. The Pivot Point has shown that is not the case. In other words, the player's expectations need to be managed. They do not need to know every bit of information in the menu systems to enjoy the game again. We can imagine a range of designs that address this issue, such as partial menu access or temporarily removing items from the player's inventory. For this and other designs, it is important to better understand player expectations. For example, while temporarily removing items from the player's inventory may seem transgressive, completing challenges 
without one's normal equipment is a common trope in games of this type (e.g. the Eventide Island quest in Breath of the Wild [15]). As designers, we can adapt these and other existing patterns from long-term single player games to serve the purpose of return.

6.1.2 Designing Sufficient Combat Exposure. Due to the constraints of modding, the Player-Designer was only able to implement four combat encounters in the quest. Combat is our most essential ingredient in progressing towards the Pivot Point, so how many combat encounters is "enough" to make the Pivot Point happen? Four combat encounters is a small amount of exposure, and more combat may be needed to reach the Pivot Point. We suggest future designs should create more open-ended combat design that enhance the exposure to combat, increasing the number of encounters and the variety of enemies that adapts to the player's Pivot Point progress, or lets players end the combat encounters once they feel ready.

6.1.3 Different Approaches To Design. We note that our design is a standalone quest, designed to be played upon a player's return to the game. We imagine that these quests could dynamically appear when the game detects a player's last save was months ago, or longer. However, this is not the only design approach to address this issue. For example, game designers could create inventory designs that are more robust to returning players in the first place. Designers could also create interventions designed for the moment of stopping play, so that the player can leave messages for their future self. Doing so could make stopping an important part of the ritual of return, as the player thinks ahead to future pleasures.

\subsection{Why Combat?}

While we worked to mitigate negative emotion and to align with expectations of the experience in our design goals, progression towards the Pivot Point is reached through combat. Long-term single player games offer a myriad of systems for players to interact with, so why does combat appear to be the most integral in the Player-Designer's Pivot Point manifestation?

In the four games we examined, combat is a primary means of interaction with the world and in the narrative, giving the player an important role in the action of the game. For example, defeating an enemy "boss" may be needed to progress in the main quest. Crucially, combat is also the source of challenge-related skill growth in the experiences. The level of the playable character increases by completing quests and lets the player expand their combat abilities through skill trees. As a result, players that have cultivated a highlevel in-game also have high-level combat abilities. Finally, combat offers a fast feedback mechanism to the player. When the player lands a hit or is attacked by an enemy, the HUD provides immediate feedback, allowing the player to evaluate how they are doing in the encounter.

Given these factors, it is not surprising that for the PlayerDesigner, combat is what makes her feel the most expert in gameplay. Successfully completing difficult combat encounters requires mechanical, cognitive and emotional focus. The association of combat with her expertise, the role it gives her in the action of the story and the world, and the emergent skill progression make combat the primary form of progression to the Pivot Point. Skilled engagement in combat helped the Player-Designer feel connected to her expert self; if she does well in combat, then the satisfaction helps her feel like her expertise is in reach.

Combat is integral to the Pivot Point experience within the context of the four games we played, yet the Pivot Point could be facilitated by different means in other games where combat is not the primary progression driver. For other games where combat isn't the primary form of interaction with the world, like Stardew Valley for instance [8], executing the goal of focusing on the primary driver of progression (design goal 2) is still intact.

\subsection{What Is the Pivot Point?}

Until now, we have discussed the Pivot Point in terms of its impact on the Player-Designer's experience. We know it is a moment of extreme breakthrough in reconnection that had monumental effects on the Player-Designer's experience, and it propelled her emotional investment forward. But what exactly is the Pivot Point? To help us in our understanding, we sought to ground the Pivot Point in existing theory. Mechanically, the Pivot Point represented a closing of the gulf of execution; cognitively, it addressed issues with memory; and emotionally, it realigned her competence with her expert self and led to emotional relief.

6.3.1 The Gulf of Execution. Norman's Gulf of Execution refers to the gap between the user's goal and the means made available by the system to achieve that goal [38]. We see it as applicable to understanding the Pivot Point. In the pre-pivot phase, the game experience did not support the Player-Designer's goal of enjoying the game and post-launch content in the same way as before she stopped playing. The struggles in the pre-pivot she experienced were such that her expectations of what she should be able to do, based on her previously cultivated expertise, and what she was able to do after time away, were incongruous. She did not have access to the mastery required by the game to play skillfully when long-term investment has been made. The Pivot Point is where the gap between the Player-Designer's perceptions of what the game experience should be like based on her previously cultivated skill, and her current abilities to execute on those perceptions begins to close. The Player-Designer is motivated to find her expert self again as a result of the Pivot Point, seeking to close the gulf of execution in the return experience to reach her expertise again.

6.3.2 Long-Term Working Memory. We also turned to psychology and different memory models to explore the Pivot Point meaning. The models of episodic and conceptual memory were of interest [9], but we were particularly intrigued by Ericsson et. al's work on long-term working memory in expert performance [16]. Long-term working memory is a theoretical model where individuals acquire memory skills needed to encode rapidly presented information in long-term memory, but they associate this information with retrieval cues kept in short term memory. The storage of domainspecific memory necessary for expert performance can be kept long term but accessed efficiently using the retrieval cues in short term memory. From this perspective, the pre-pivot experiences highlight that the retrieval mechanisms were not in short term memory due to time away, but the encoded information in long term memory became accessible at the Pivot Point due to a retrieval cue becoming active again. This could explain why the Player-Designer was so 


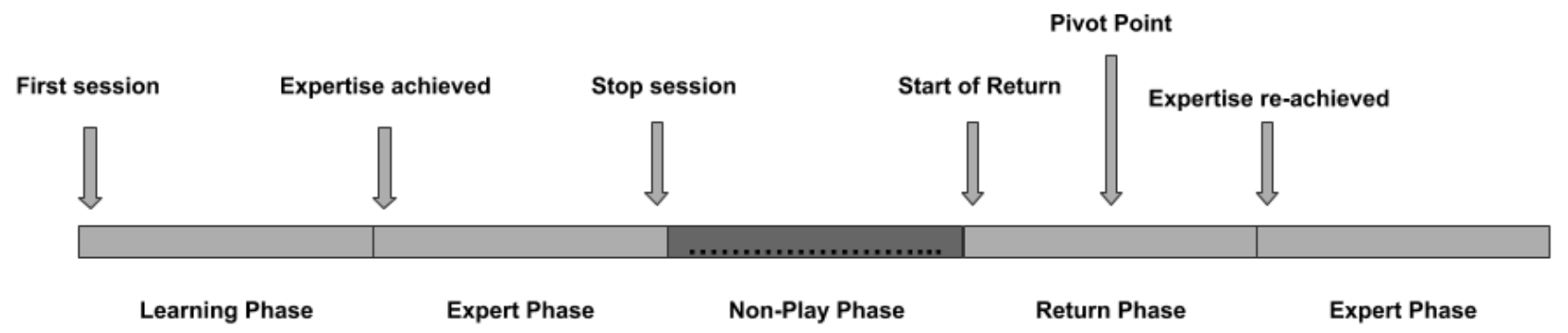

Figure 7: The Player-Designer's journey of play of a long-term single player game, from the first time she started playing, to the return experience and beyond

synchronized with her expert self in that encounter despite the pre-pivot struggles with mechanical competence.

6.3.3 Competence. The psychological needs of autonomy, competence and relatedness are essential to a game experience. For our purposes we are specifically interested in competence and its connection to the Pivot Point. Competence as it relates to Self Determination Theory is the feeling of effectance and capability in the face of challenge [43]. It leads to a feeling of mastery on the player's part, and is an important contributor to enjoyment in games [47]. The Pivot Point is where the Player-Designer felt competent during an optimally difficult combat encounter, as in it required her expert skill to be enabled, which led to her feeling her expert self was in reach. Moreover, it is not only that she was able to defeat the encounter; it was how she did it. She won the fight expertly and with finesse, reclaiming the competence of her previous expert self. The competence the Player-Designer feels then is relative to her previous self's expertise; it was only when she felt competent as her expert self once did that she began enjoying the experience again. This relative competence could help explain the emotional effects of the return in the pre- and post-pivot stages. Initially, she felt less competent compared to her previous self, adding to the "embarrassment" she felt at not being able to play the game as well as she used to. The Pivot Point triggers an emotional relief postpivot, and knowing that her competence is still in reach, she began enjoying the experience again.

6.3.4 Situating The Pivot Point in The Gameplay Journey. The Pivot Point and the associated pre and post-pivot experiences are all part of the Player-Designer's process towards recovering expertise and full re-engagement. These three elements form a return phase. In the context of the full long-term single player game experience, we can visualize this phase as part of the Player-Designer's entire journey in playing the game (Figure 7). At the start of the journey of play, the Player-Designer plays the game for the first time (First Session) from the beginning, learning how to play via tutorials and iterative exposure designed to introduce her to the game world. While investing extended hours of play time, upwards of one hundred hours, she gains expertise (Expertise achieved) and enters the Expert Phase of play.
Due to life circumstances or a desire to move on to other games, the Player-Designer drops the game, and her final session of play is the Stop session. Months or years after moving on from the game (Non-Play Phase), a new DLC is released that continues the story of the original game, and is only accessible after completing the main storyline of the base game. This entices the Player-Designer to return to the game. She starts the return journey (Start of Return) and enters the Return Phase, consisting of the three pivot stages we have identified. In getting through the various mechanical, cognitive and emotional struggles of the pre-pivot, the Player-Designer reaches the Pivot Point, which is not a snapshot in time but rather a process in a combat encounter that propels her to the post-pivot enjoyment. Should post-pivot progress towards recovering expertise continue, full re-engagement will occur (Expertise re-achieved) and the PlayerDesigner once again returns to the Expert Phase.

\section{LIMITATIONS}

Given how understudied this domain is, we sought the first-hand experience to ground our future explorations. As a result of our work here, we have a clearer understanding of the problem, potential issues players might face, and a design pathway that directly emerged from our return experiment. While there are interesting implications and future avenues that resulted, there are multiple limitations that must be highlighted and addressed in these future avenues of exploration.

While autobiographical design and associated first-person methods helped us better understand return effects and the Pivot Point from a first-person perspective, the results are inherently tied to a single participant; the Player-Designer. The Player-Designer's return experiences, the discovery of The Pivot Point, and the proposed designs cannot be immediately generalized to the broader set of players. Moreover, the Player-Designer took part in analyzing data from her own experiences, which introduces bias in our analysis.

Our work here is limited to returns in long-term single player games. We also did not exhaustively cover all aspects of Player Experience (PX), such as immersion and flow. Another limitation was the short play sessions. While three and a half hour play sessions 
were reasonable considering the length of most post-launch content, we found that the Player-Designer could not return to full game skill and enjoyment in that length of time. We do not yet know how long it takes for expertise to fully return, nor how the proposed designs affect the experience. The Player-Designer could not evaluate the created designs, and how they influence the Pivot Point, in time for publication. These lingering questions can be addressed in future studies.

\section{FUTURE WORK}

While our results are autobiographical, our understanding of return effects and the Pivot Point present an exciting and powerful opportunity to progress in the problem domain and design better return experiences. The limitations introduced by the first-person methods we employed can be addressed by executing studies with users outside of the research team, which will be our immediate step forward in the project. Working with a greater cross section of players will allow us to examine the Pivot Point manifestation and deepen our understanding of returns to long-term single player games, and the designs needed to support such returns.

Situating and examining the work more closely within existing games research will provide further opportunities of exploration. The future studies with other participants should involve more varied PX measures and longer play sessions that will enrich the dataset. Beyond introducing PX methodological practices, we are also interested in leveraging Andersen et. al and Cheung et. al's work on game tutorials and the first hour experience $[1,7]$ to deepen Pivot Point understanding and inform return experience design.

Our use of the first-person methods have shown promise, and we are optimistic about the continued use and evolution of methods such as autoethnography and autobiographical design in games and broader HCI research. We acknowledge that our work makes use of only two first-person research approaches. Future explorations of returns can expand the breadth of first-person methods to include fully fledged autoethnographies, as well as studies making use of more critical first-person methods such as close reading [2].

The investment required for expertise in different genres of games, such as multiplayer first-person shooters, is different than that of the long-term single player games we examined. Thus, along with examining Pivot Point manifestation in other players, studies must be conducted to explore returns and Pivot Points in other game genres beyond long-term single player games.

The exploration of returning to long-term single player games is the first instance of studying returns and expertise rediscovery in video games. However, it carries implications for the broader $\mathrm{HCI}$ community as well. Complex tasks such as software development and video editing require long-term investment from users into cultivating expertise and fluency. Understanding how this fluency and expertise is affected when returning after time away presents further avenues of exploration of Pivot Point manifestation.

\section{CONCLUSION}

In this paper, we set out on the path to begin understanding return experiences in long-term single player games. We utilized an autobiographical design approach to experience returns to immerse ourselves in the problem space, where the Player-Designer experienced extreme struggles but also extreme breakthroughs. The Player-Designer underwent four returns that elucidated the Pivot Point across all return experiences we examined, which drove the subsequent design explorations. Through the prototyping work the Player-Designer conducted, we learned how focusing on combat, reducing negative emotion, and managing player expectations can help us with facilitating the Pivot Point. As a result of the autobiographical journey, we now have a better understanding of how to move forward in this problem space, and we see the Pivot Point as a viable path in learning how to design better video game returns beyond the autobiographical scope of our work here. Our future studies will work to find generalizable observations about the long-term single player return experience that our autobiographical design journey cannot capture, along with examining and studying how the Pivot Point manifestation occurs in other players.

\section{ACKNOWLEDGMENTS}

We are grateful for the opportunity to use the commercial games developed by Ubisoft Quebec, CD Projekt Red, Guerilla Games and Santa Monica Studio. We would also like to thank the modding community of The Witcher 3: Wild Hunt, particularly those belonging to the Radish QuestUI Discord. Finally, we would like to thank our reviewers for their constructive feedback. This work is funded by the Natural Sciences and Engineering Council of Canada (NSERC).

\section{REFERENCES}

[1] Erik Andersen, Eleanor O’Rourke, Yun-En Liu, Rich Snider, Jeff Lowdermilk, David Truong, Seth Cooper, and Zoran Popovic. 2012. The Impact of Tutorials on Games of Varying Complexity. In Proceedings of the SIGCHI Conference on Human Factors in Computing Systems (Austin, Texas, USA) (CHI '12). Association for Computing Machinery, New York, NY, USA, 59-68. https://doi.org/10.1145/ 2207676.2207687

[2] Jim Bizzocchi and Joshua Tanenbaum. 2011. Well read: Applying close reading techniques to gameplay experiences. ETC Press, Pittsburgh, PA, USA, Chapter 23, 289-313.

[3] Fran C. Blumberg, Sheryl F. Rosenthal, and John D. Randall. 2008. Impasse-Driven Learning in the Context of Video Games. Comput. Hum. Behav. 24, 4 (July 2008), 1530-1541. https://doi.org/10.1016/j.chb.2007.05.010

[4] Daniel Van Boom. 2019. PS4, Xbox One and Nintendo Switch: This generation's best-selling games in the US so far. https://www.cnet.com/news/ps4-xbox-oneand-nintendo-switch-this-generations-best-selling-games-in-the-us-so-far/

[5] Bill Buxton. 2010. Sketching user experiences: getting the design right and the right design. Morgan kaufmann, Burlington, MA, USA

[6] Mark G. Chen. 2009. Communication, Coordination, and Camaraderie in World of Warcraft. Games and Culture 4, 1 (2009), 47-73. https://doi.org/10.1177/ 1555412008325478

[7] Gifford K. Cheung, Thomas Zimmermann, and Nachiappan Nagappan. 2014. The First Hour Experience: How the Initial Play Can Engage (or Lose) New Players. In Proceedings of the First ACM SIGCHI Annual Symposium on Computer-Human Interaction in Play (Toronto, Ontario, Canada) (CHI PLAY '14). Association for Computing Machinery, New York, NY, USA, 57-66. https://doi.org/10.1145/ 2658537.2658540

[8] ConcernedApe. 2016. Stardew Valley. Game [PC]. ConcernedApe.

[9] Martin A. Conway. 2009. Episodic memories. Neuropsychologia 47, 11 (2009), 2305 - 2313. https://doi.org/10.1016/j.neuropsychologia.2009.02.003 Episodic Memory and the Brain.

[10] Jeff Cork. 2018. How Assassin's Creed Odyssey Is Doubling Down On Its RPG Elements. https://www.gameinformer.com/e3-2018/2018/06/11/how-assassinscreed-odyssey-is-doubling-down-on-its-rpg-elements

[11] Thomas Debeauvais, Bonnie Nardi, Diane J. Schiano, Nicolas Ducheneaut, and Nicholas Yee. 2011. If You Build It They Might Stay: Retention Mechanisms in World of Warcraft. In Proceedings of the 6th International Conference on Foundations of Digital Games (Bordeaux, France) (FDG '11). Association for Computing Machinery, New York, NY, USA, 180-187. https://doi.org/10.1145/2159365. 2159390 
[12] Audrey Desjardins and Aubree Ball. 2018. Revealing Tensions in Autobiographical Design in HCI. In Proceedings of the 2018 Designing Interactive Systems Conference (Hong Kong, China) (DIS '18). Association for Computing Machinery, New York, NY, USA, 753-764. https://doi.org/10.1145/3196709.3196781

[13] Tapajit Dey, Jacob Logan Massengill, and Audris Mockus. 2016. Analysis of Popularity of Game Mods: A Case Study. In Proceedings of the 2016 Annual Symposium on Computer-Human Interaction in Play Companion Extended Abstracts (Austin, Texas, USA) (CHI PLAY Companion '16). Association for Computing Machinery, New York, NY, USA, 133-139. https://doi.org/10.1145/2968120.2987724

[14] Katharina Emmerich, Patrizia Ring, and Maic Masuch. 2018. I'm Glad You Are on My Side: How to Design Compelling Game Companions. In Proceedings of the 2018 Annual Symposium on Computer-Human Interaction in Play (Melbourne, VIC, Australia) (CHI PLAY '18). Association for Computing Machinery, New York, NY, USA, 141-152. https://doi.org/10.1145/3242671.3242709

[15] Nintendo EPD. 2017. The Legend of Zelda: Breath of the Wild. Game [Nintendo Switch]. Nintendo.

[16] K. Anders Ericsson, Robert R. Hoffman, Aaron Kosbelt, and A. Mark Williams. 2018. The Cambridge Handbook of Expertise and Expert Performance (2 ed.) Cambridge University Press, New York, NY, USA. https://doi.org/10.1017/ 9781316480748

[17] Alessandro Febretti and Franca Garzotto. 2009. Usability, Playability, and LongTerm Engagement in Computer Games. In CHI '09 Extended Abstracts on Human Factors in Computing Systems (Boston, MA, USA) (CHI EA '09). Association for Computing Machinery, New York, NY, USA, 4063-4068. https://doi.org/10.1145/ 1520340.1520618

[18] Jesse Fox and Wai Yen Tang. 2017. Women's experiences with general and sexual harassment in online video games: Rumination, organizational responsiveness, withdrawal, and coping strategies. New Media \& Society 19, 8 (2017), 1290-1307.

[19] Allegra Frank. 2018. RPGs need in-game recaps to help us out. https://www. polygon.com/2018/7/31/17607734/rpg-in-game-recap-backlog

[20] Guerilla Games. 2017. Horizon:Zero Dawn. Game [PlayStation 4]. Sony Interactive Entertainment, Last Played August 2019.

[21] Chaim Gartenberg. 2018. Assassin's Creed: Odyssey is even more of a traditional RPG than last year's Origins. https:/www.theverge.com/2018/6/11/17430878/ assassins-creed-odyssey-hands-on-e3-2018-rpg-ubisoft

[22] James Paul Gee. 2003. What Video Games Have to Teach Us about Learning and Literacy. Comput. Entertain. 1, 1 (Oct. 2003), 20. https://doi.org/10.1145/950566. 950595

[23] James Paul Gee. 2007. Good video games+ good learning: Collected essays on video games, learning, and literacy. Peter Lang, Bern, Switzerland.

[24] Noor Hammad, Elaheh Sanoubari, Patrick Finn, Sowmya Somanath, James E. Young, and Ehud Sharlin. 2019. Mutation: Leveraging Performing Arts Practices in Cyborg Transitioning. In Proceedings of the 2019 on Creativity and Cognition (San Diego, CA, USA) (C\&C '19). Association for Computing Machinery, New York, NY, USA, 53-59. https://doi.org/10.1145/3325480.3325508

[25] Robin Hunicke, Marc LeBlanc, and Robert Zubek. 2004. MDA: A formal approach to game design and game research. In Proceedings of the AAAI Workshop on Challenges in Game AI, Vol. 4. Game Developers Conference, San Jose, CA, USA, 1722 .

[26] Ioanna Iacovides, Anna L. Cox, Ara Avakian, and Thomas Knoll. 2014. Player Strategies: Achieving Breakthroughs and Progressing in Single-Player and Cooperative Games. In Proceedings of the First ACM SIGCHI Annual Symposium on Computer-Human Interaction in Play (Toronto, Ontario, Canada) (CHI PLAY '14). Association for Computing Machinery, New York, NY, USA, 131-140. https://doi.org/10.1145/2658537.2658697

[27] Ioanna Iacovides, Anna L Cox, Patrick McAndrew, James Aczel, and Eileen Scan lon. 2015. Game-play breakdowns and breakthroughs: exploring the relationship between action, understanding, and involvement. Human-computer interaction 30, 3-4 (2015), 202-231.

[28] Fares Kayali, Naemi Luckner, Peter Purgathofer, Katta Spiel, and Geraldine Fitzpatrick. 2018. Design Considerations towards Long-Term Engagement in Games for Health. In Proceedings of the 13th International Conference on the Foundations of Digital Games (Malmö, Sweden) (FDG '18). Association for Computing Machinery, New York, NY, USA, Article 35, 8 pages. https://doi.org/10.1145/3235765.3235789
[29] Elizabeth Keating and Chiho Sunakawa. 2010. Participation cues: Coordinating activity and collaboration in complex online gaming worlds. Language in Society 39, 3 (2010), 331-356. https://doi.org/10.1017/S0047404510000217

[30] Amrita Khalid. 2020. Why do studios release such long video games? https: //qz.com/1787043/why-are-video-games-getting-longer/

[31] Jarrod Knibbe, Jonas Schjerlund, Mathias Petraeus, and Kasper Hornbæk. 2018. The Dream is Collapsing: The Experience of Exiting VR. In Proceedings of the 2018 CHI Conference on Human Factors in Computing Systems (Montreal OC, Canada) (CHI '18). Association for Computing Machinery, New York, NY, USA, 1-13. https://doi.org/10.1145/3173574.3174057

[32] Andrés Lucero. 2018. Living Without a Mobile Phone: An Autoethnography. In Proceedings of the 2018 Designing Interactive Systems Conference (Hong Kong, China) (DIS '18). Association for Computing Machinery, New York, NY, USA, 765-776. https://doi.org/10.1145/3196709.3196731

[33] Andrés Lucero, Audrey Desjardins, Carman Neustaedter, Kristina Höök, Marc Hassenzahl, and Marta E. Cecchinato. 2019. A Sample of One: First-Person Research Methods in HCI. In Companion Publication of the 2019 on Designing Interactive Systems Conference 2019 Companion (San Diego, CA, USA) (DIS '19 Companion). Association for Computing Machinery, New York, NY, USA, 385-388. https://doi.org/10.1145/3301019.3319996

[34] Lavinia McLean and Mark D Griffiths. 2019. Female gamers' experience of online harassment and social support in online gaming: a qualitative study. International fournal of Mental Health and Addiction 17, 4 (2019), 970-994.

[35] David A. Mellis and Leah Buechley. 2014. Do-It-Yourself Cellphones: An Investigation into the Possibilities and Limits of High-Tech Diy. In Proceedings of the SIGCHI Conference on Human Factors in Computing Systems (Toronto, Ontario, Canada) (CHI '14). Association for Computing Machinery, New York, NY, USA, 1723-1732. https://doi.org/10.1145/2556288.2557309

[36] Carman Neustaedter and Phoebe Sengers. 2012. Autobiographical Design in HCI Research: Designing and Learning through Use-It-Yourself. In Proceedings of the Designing Interactive Systems Conference (Newcastle Upon Tyne, United Kingdom) (DIS '12). Association for Computing Machinery, New York, NY, USA, 514-523. https://doi.org/10.1145/2317956.2318034

[37] Carman Neustaedter and Phoebe Sengers. 2012. Autobiographical design: What you can learn from designing for yourself. interactions 19 (11 2012), 28-33. https://doi.org/10.1145/2377783.2377791

[38] Don Norman. 2013. The design of everyday things: Revised and expanded edition. Basic books, New York, NY, USA.

[39] Bruce Phillips. 2009. Quitter Talk. https://www.gamasutra.com/view/feature/ 4171/staying_power_rethinking_feedback_php?print $=1$

[40] Ubisoft Quebec. 2018. Assassin's Creed: Odyssey. Game [PlayStation 4]. Ubisoft, Last Played August 2019.

[41] Amon Rapp. 2018. Autoethnography in Human-Computer Interaction: Theory and Practice. Springer International Publishing, Cham, 25-42. https://doi.org/10. 1007/978-3-319-73374-6 3

[42] CD Projekt Red. 2015. The Witcher 3: Wild Hunt. Game [PlayStation 4]. Warner Bros Interactive Entertainment, Last Played August 2020.

[43] Richard M Ryan, C Scott Rigby, and Andrew Przybylski. 2006. The motivational pull of video games: A self-determination theory approach. Motivation and emotion 30, 4 (2006), 344-360.

[44] Björn Strååt and Harko Verhagen. 2018. Exploring Video Game Design and Player Retention- a Longitudinal Case Study. In Proceedings of the 22nd International Academic Mindtrek Conference (Tampere, Finland) (Mindtrek '18). Association for Computing Machinery, New York, NY, USA, 39-48. https://doi.org/10.1145/ 3275116.3275140

[45] Santa Monica Studio. 2018. God of War. Game [PlayStation 4]. Sony Interactive Entertainment, Last Played August 2019.

[46] Carl Therrien. 2013. Immersion. Routledge, Abingdon, England; New York, NY, Chapter 55, 451-459. https://doi.org/10.4324/9780203114261.ch55

[47] Ahmet Uysal and Irem Yildirim. 2016. Self-Determination Theory in Digital Games. Springer, Cham, New York, NY, USA, 123-135. https://doi.org/10.1007/978-3319-29904-4_8

[48] Zack Zwiezen. 2020. Running Around During Loading Screens Is An Important Part Of Assassin's Creed. https://kotaku.com/runningaround-during-loading-screens-is-an-important-p-1844170039\#: : text=Loadingscreensoftensuck., has(mostly)stuckaround. 